\section{Microscopy}

Coming Events

Due to COVID-19, please check to see if the listed events have been postponed or cancelled.

\section{0}

APT\&M 2020 - Atom Probe

Tomography \& Microscopy

July $7-10,2020$

Oxford, England

https://aptm2020.web.ox.ac.uk

Microscopy \& Microanalysis 2020

August 2-6, 2020

Milwaukee, WI

www.microscopy.org

16th International Congress of Histochemistry and Cytochemistry (ICHC)

August 30-September 2, 2020

Prague, Czech Republic

http://ichc2020.com

Neuroscience 2020

October 24-28, 2020

Washington, DC

www.sfn.org/meetings/neuroscience-2020

\section{MRS Fall Meeting \& Exhibit}

November 29-December 4, 2020

Boston, MA

www.mrs.org/fall2020

\section{ASCB 2020 Annual Meeting}

December 5-9, 2020

Philadelphia, PA

www.ascb.org/meetings-events/future-ascb-meetings

\section{1}

Microscopy \& Microanalysis 2021

August 1-5, 2021

Pittsburgh, PA

www.microscopy.org

\section{2}

Microscopy \& Microanalysis 2022

July 31-August 4, 2022

Portland, OR

www.microscopy.org

\section{3}

Microscopy \& Microanalysis 2023

July 24-28, 2023

Minneapolis, MN

www.microscopy.org

\section{4}

Microscopy \& Microanalysis 2024

July 28-August 1, 2024

Cleveland, $\mathrm{OH}$

www.microscopy.org

\title{
Visualizing Vesicles as Biomarkers in Cancer
}

\author{
Stephen W. Carmichael \\ Mayo Clinic, Rochester, MN 55905 \\ carmichael.stephen@mayo.edu
}

Advances in optical imaging technologies have a way of revealing structures in new ways, seeing things in a new light, and providing new information about fundamental biological processes such as carcinogenesis. Two of the factors driving these technologies are new laser sources and new ways of pulse-shaping or manipulating light to enhance nonlinear optical imaging processes in tissues. Recent work has focused on using lower repetition-rate ultrafast lasers to pump photonic crystal fibers to generate stable, low-noise, widely coherent supercontinuum that can then be used to excite multiple nonlinear processes in tissues. Simultaneous label-free autofluorescence multi-harmonic (SLAM) microscopy was recently demonstrated to generate two- and three-photon autofluorescence and second- and third-harmonic generation imaging channels simultaneously, enabling structural, molecular, and metabolic imaging in

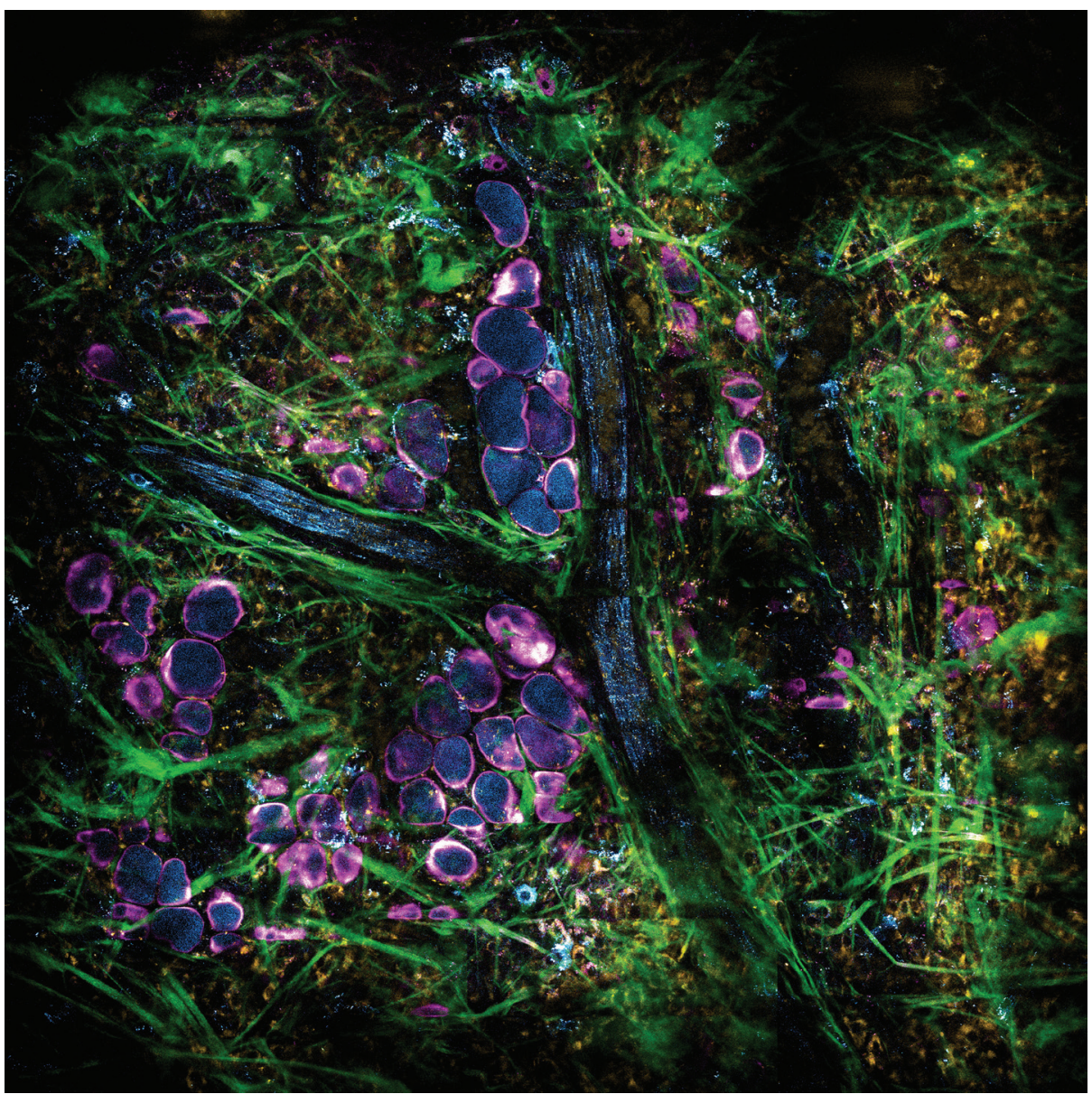

Figure 1: Label-free SLAM microscopy image of a mammary tumor. The multimodal color channels include: Green - Second Harmonic Generation from collagen; Magenta - Third Harmonic Generation from interfaces, such as between lipid/aqueous compartments; Yellow - Two-Photon Excited Autofluorescence from FAD; Blue - Three-Photon Excited Autofluorescence from NAD $(\mathrm{P}) \mathrm{H}$. Extracellular vesicles appear primarily as blue/magenta single-pixel objects due to their $\mathrm{NAD}(\mathrm{P}) \mathrm{H}$ content and size. 


\section{hORIBA Scientific}

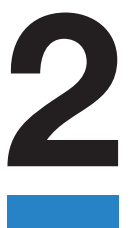

NEW

Innovative

Microscopy

Products from $\mathrm{HOR} \mid \mathrm{BA}$

Applications like

FLIM, TRPL,

photoluminescence,

Raman and

electroluminescence

require more

capabilities than your microscope can provide.

HORIBA has two new

products to help you

multitask your microscope.
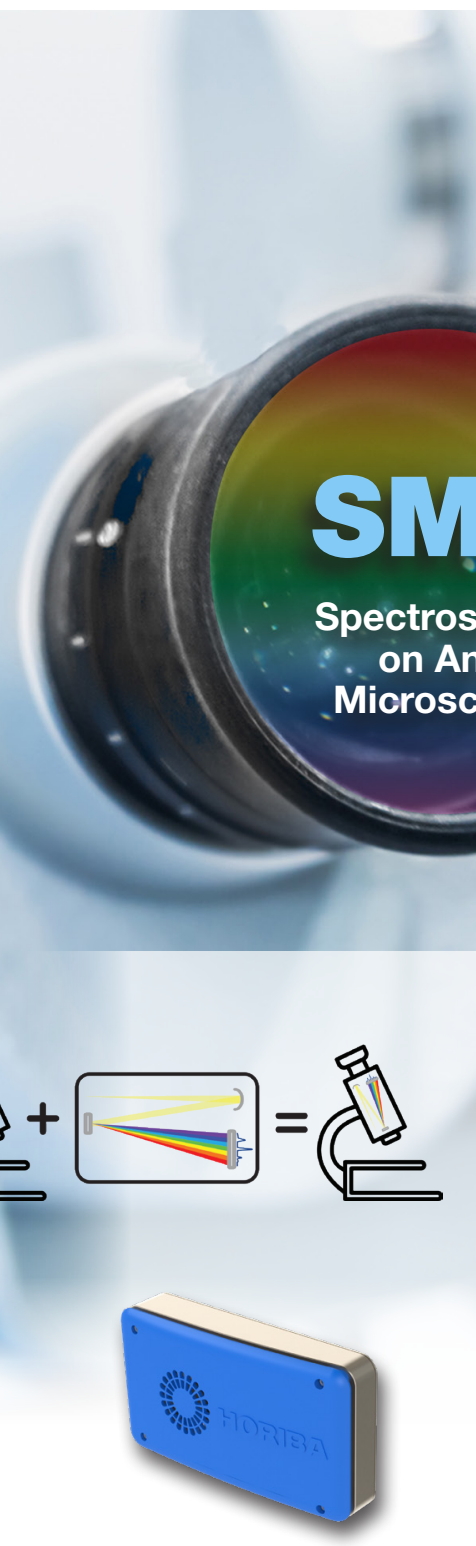

NEW Standard Microscope Systems

Add various spectroscopies to

ANY STANDARD Microscope

without sacrificing performance of either one!

\section{NEW SPAD FLIMera Camera}

Fluorescence lifetime images from

25K simultaneous pixel measurements

at a video rate of 30 frames per second!

Find out more at: horiba.com/microscopy 
real time. Importantly, this is label-free, as labels may alter the biology. SLAM microscopy was recognized as one of the recipients of the Microscopy Today Innovation Awards for 2019.

Historically, advances in imaging have enabled investigators to visualize things in a novel manner, and therefore ask new questions that were previously not conceivable. Recently the group headed by Stephen Boppart, the same group that developed SLAM microscopy, has taken the technology a step futher. In a publication by Sixian You, Stephen Boppart and others showed that it was possible to visualize extracellular vesicles (EVs) in tissues and in vivo without the use of dyes, probes, or other labels [1]. The most exciting part is that this may have clinical applications. EVs is a collective term for exosomes, microvesicles, and apoptotic bodies. These cell-derived particles have generated extensive interest as we learn more about how cells use them for intercellular communication in states of health and disease, particularly in cancer. It is known that cancer cells rapidly increase the production of EVs early in the disease, well before solid tumors appear. Therefore, it is thought that these EVs are important in the progression and spread of cancer. It is hypothesized that EVs released from cancer cells circulate throughout the body early in carcinogenesis, are taken up by normal cells, and direct them to upregulate their metabolism. This could facilitate the body to host the growing and spreading tumor.

Whereas much remains to be elucidated about EVs, it is exciting that SLAM microscopy is able to detect and visualize the presence and spatial distribution of EVs in tissue specimens and in vivo. The recent paper by You et al., with their multimodal optical imaging approach, identified unique optical signatures in breast cancer. They also showed that these signatures of EVs can be used to differentiate tumor-associated EVs from normal EVs and also indicate the aggressiveness of the tumor (Figure 1). They think that the autofluorescence generated by nicotinamide adenine dinucleotide phosphate (NADPH) within the EVs, as well as the size and structural composition of the EV membrane, in turn generates a strong third-harmonic signal between lipid-aqueous interfaces. You et al. showed that these signatures from the EVs coming from various breast cancer (and normal) cell lines from rat mammary tumors and from human breast cancer specimens indicate the tumor when imaging during surgery. They also found signatures for normal EVs in breast tissues from normal patients.

Whereas SLAM microscopy has generated stunning and data-rich images, there also exists the potential for using these unique multi-modal optical signatures as biomarkers for both clinical diagnosis and for screening. Ongoing efforts by this team may lead to a way of detecting cancer in urine samples [2]. This potentially will shed new light on liquid biopsies for cancer!

\section{References}

[1] S You et al., Proc Nat Acad Sci 116(48) (2019) 24012-18, www.pnas.org/cgi/doi/10.1073/pnas.1909243116.

[2] S Boppart, personal communication.

[3] The author gratefully acknowledges Dr. Stephen Boppart for extensive assistance with this article.

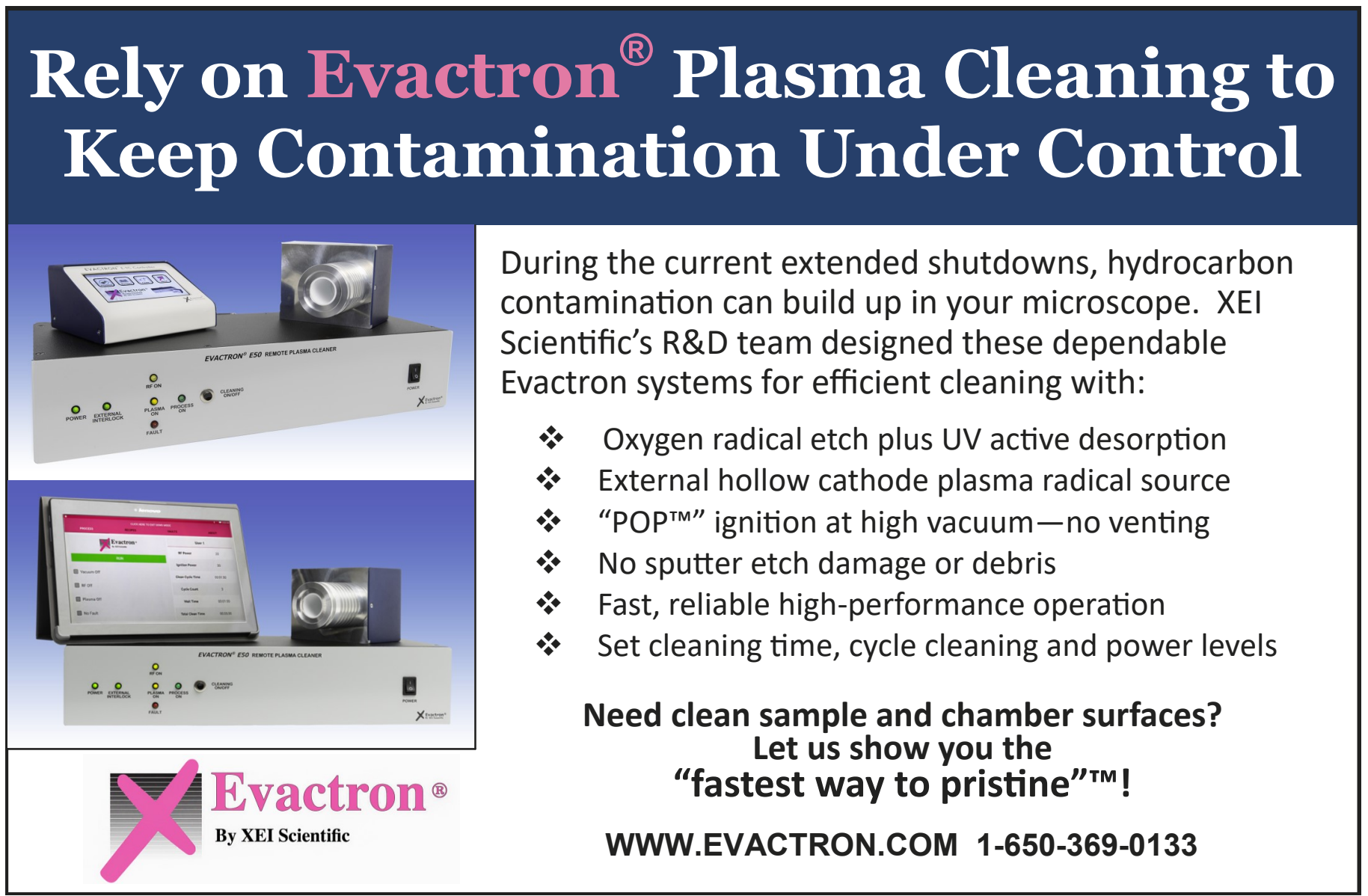




\section{First $\gg \gg\rangle$}

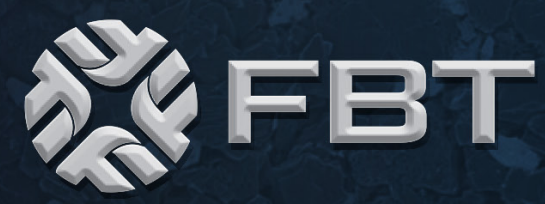

\section{HIGH THROUGHPUT SEM System}

\section{Navigator-10T}

(6) times faster than
conventional FE SEMs

Direct electron detection technology and Dual channel imaging (SE\&BSE) Intelligent atlas and cross-scale

(国 Big data intelligent analysis and Al computing

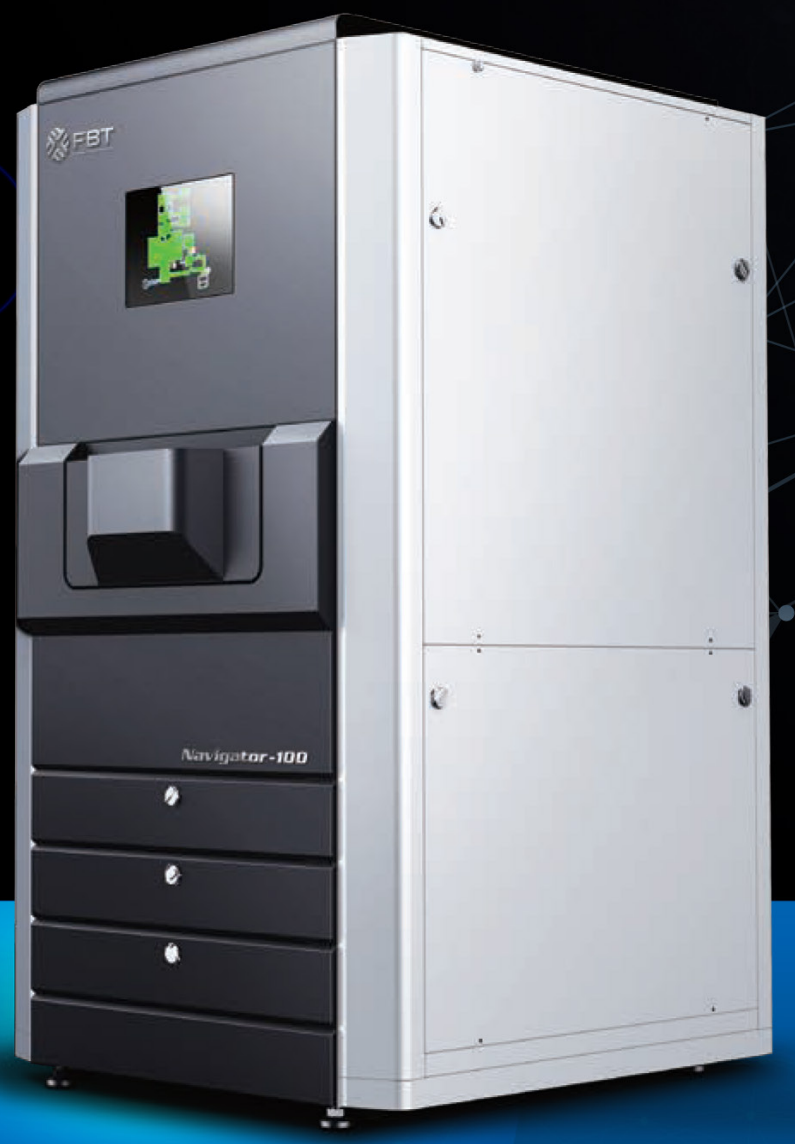

CHINA

FOCUS EBEAM TECHNOLOGY(BEIJING) CO. ,LTD

Tel: +86 01067832505 ext 813 